\title{
Quantitative and qualitative analysis of asynchronous neural activity
}

\author{
Ekkehard Ullner (1) and Antonio Politi 10 \\ Institute for Pure and Applied Mathematics and Department of Physics (SUPA), Old Aberdeen, Aberdeen AB24 3UE, United Kingdom \\ Alessandro Torcini $\odot$ \\ Laboratoire de Physique Théorique et Modélisation, Université de Cergy-Pontoise, CNRS, UMR 8089, 95302 Cergy-Pontoise cedex, France \\ and Istituto dei Sistemi Complessi, CNR_Consiglio Nazionale delle Ricerche, via Madonna del Piano 10, I-50019 Sesto Fiorentino, Italy
}

(Received 17 December 2019; accepted 25 March 2020; published 29 April 2020)

\begin{abstract}
The activity of a sparse network of leaky integrate-and-fire neurons is carefully revisited with reference to a regime of bona fide asynchronous dynamics. The study is preceded by a finite-size scaling analysis, carried out to identify a setup where collective synchronization is negligible. The comparison between quenched and annealed networks reveals the emergence of substantial differences when the coupling strength is increased, via a scenario somehow reminiscent of a phase transition. For sufficiently strong synaptic coupling, quenched networks exhibit a highly bursting neural activity, well reproduced by a self-consistent approach, based on the assumption that the input synaptic current is the superposition of independent renewal processes. The distribution of interspike intervals turns out to be relatively long-tailed; a crucial feature required for the self-sustainment of the bursting activity in a regime where neurons operate, on average, (much) below threshold. A semiquantitative analogy with Ornstein-Uhlenbeck processes helps validate this interpretation. Finally, an alternative explanation in terms of Poisson processes is offered under the additional assumption of mutual correlations among excitatory and inhibitory spikes.
\end{abstract}

DOI: 10.1103/PhysRevResearch.2.023103

\section{INTRODUCTION}

The characterization of the spiking activity of neuronal networks is a long-standing problem even with reference to the asynchronous regime: simple from a dynamical point of view, but extremely relevant for understanding cortex dynamics $[1,2]$. A moment's reflection indeed suggests that this is not a trivial task whenever the self-generated input current is not constant: To what extent can the deterministic fluctuations be treated as a stochastic process?

Generally, the problem is tackled by assimilating the input current to a $\delta$-correlated Gaussian process [3] and thereby deriving a self-consistent Fokker-Planck equation. Under the additional simplifying assumption of a piecewise linear dynamics [as in leaky integrate-and-fire (LIF) neurons], a solution can be analytically determined and its stability assessed [4].

Although this approach turns out to be relatively accurate for small coupling strengths, the same is no longer true for stronger coupling (see Ref. [5]), when large deviations from the theoretical predictions are observed. These deviations may in principle originate from various sources: (i) the spontaneous onset of irregular collective dynamics, which emerges

Published by the American Physical Society under the terms of the Creative Commons Attribution 4.0 International license. Further distribution of this work must maintain attribution to the author(s) and the published article's title, journal citation, and DOI. even for relatively small network connectivities [5,6]; (ii) the non-Poissonian nature of the spiking activity; (iii) large amplitude of the single spikes and the consequential possible failure of a perturbative, linear approach; and (iv) the presence of non-negligible finite-time correlations.

Several alternative approaches have indeed been proposed. For instance, an exact treatment of shot noise for spike amplitudes not vanishingly small, which leads to a mixed FokkerPlanck/master-equation formalism (see Refs. [7,8]). Unfortunately, we are not aware of any way to make the approach self-consistent, by inferring the input properties on the basis of the observed output. Anyhow, since this approach assumes a Poissonian distribution of the interspike intervals (ISIs) - a property largely unsatisfied for strong synaptic coupling-one should look for different approximation schemes anyway.

A different strategy was proposed by Dummer et al. [9], based on the self-consistent derivation of the power spectrum of the spiking activity. The advantage of this method is that no assumption is made on the spectral shape of the synaptic current. While the original implementation already proved unstable for relatively small coupling strengths, the variant recently proposed in Ref. [10] leads to a seemingly accurate reproduction of the network dynamics. We shall treat it as a reference for some of our considerations.

In this paper, we revisit the problem of characterizing the asynchronous regime in the context of two coupled populations of excitatory and inhibitory LIF neurons accompanied by refractoriness and delay $[4,11,12]$. In spite of the restriction to a specific type of neurons (motivated by the opportunity to compare with many numerical and analytical studies), the 
approach we propose is general and can be implemented regardless of the single-neuron evolution equation.

The first goal is to determine the minimal network size such that the numerical results can be considered as truly asymptotic. This is achieved by first checking the accuracy of numerical simulations and then estimating finite-size corrections. In Refs. [5,6], it was indeed shown that the firing activity of a network of 10000 LIF neurons with an in-degree $K=1000$ is strongly affected by the presence of collective irregular dynamics.

To clarify the role played by the different "ingredients," we introduce a second setup: annealed networks, where postsynaptic neighbors are randomly selected whenever a new spike is emitted. From a biological point of view, this setup can be justified by recalling that synaptic transmission in the cortex is highly unrealiable: On average, at room temperature, most synapses respond to only $\simeq 13 \%$ of the the presynaptic spikes [13]. Unreliable synapses are usually analyzed at either the single-unit level [14], or of neural populations [15] to understand how this unreliability actually affects information transmission. Moreover, from a statistical-mechanics point of view, this choice will help in better understanding the dynamics of the system, since it is known that quenched and annealed systems may exhibit different properties from one another [16]. Interestingly, this happens in the present model above a "critical" synaptic coupling strength (namely, $J \approx$ 0.25 ). The existence of two seemingly different phases was already claimed by Ostojic [11], but challenged in Ref. [17]. Here, however, we do not investigate the behavior in the vicinity of the hypothetical phase transition, but rather focus on the large-coupling regime, since it is more important to first characterize the different phases which may emerge upon varying the coupling strength.

One of the main results of this paper is that the synaptic current can be accurately represented as the superposition of independent identical renewal processes (RPs), each characterized by a suitable ISI distribution. We also show that the correlations due to the long-tailed ISI distribution can be equivalently represented as long-term memory in the symbolic representation of inhibitory versus excitatory spikes.

More precisely, in Sec. II, we introduce the model and define the indicators later used to characterize and discuss the various dynamical properties. In the following Sec. III, we illustrate the firing activity of the quenched network, computing several indicators for different coupling strengths. A relatively quick discussion is also devoted to the annealed set-up to show the differences with respect to the quenched case. In Sec. IV, we first introduce the two self-consistent approaches herein implemented to characterize the neural activity. The former one, based on the distribution of ISIs, provides a rather accurate description. The latter, already proposed in Refs. $[9,10]$, reveals an unexpectedly stable fixed point, which, however, is further away from the results of accurate simulations. In Sec. V, we turn our attention to the bursting activity observed for large coupling in an attempt to explain how neurons operating, on average, below threshold are able to exhibit a strong firing activity. Finally, in Sec. VI we summarize the main results and focus on the still open problems.

\section{MODEL AND METHODS}

\section{A. Network model}

Due to its relevance in the context of asynchronous dynamics in balanced networks $[4,11,18,19]$, we consider the following sparse spiking network of LIF neurons. The network is composed of $b N$ excitatory and $(1-b) N$ inhibitory cells; the membrane potential $V_{i}$ of the $i$ th neuron evolves according to the equation

$$
\tau \dot{V}_{i}=R\left(I_{0}+I_{i}\right)-V_{i},
$$

where $\tau=20 \mathrm{~ms}$ is the membrane time constant, $R I_{0}=$ $24 \mathrm{mV}$ is an external DC supra-threshold "current," while $R I_{i}$ is the synaptic current arising from the recurrent coupling

$$
R I_{i}(t)=\tau J \sum_{n} G_{i j(n)} \delta\left(t-t_{n}^{(j)}-\tau_{d}\right),
$$

where $J$ is the coupling strength and the sum runs over all the spikes emitted at time $t_{n}^{(j)}<t$ from the presynaptic neurons $j(n)$ connected to neuron $i$. $G_{i j}$ is the adjacency matrix and its elements assume the following values: $G_{i j}=1(-g)$, if the presynaptic neuron $j$ is excitatory (inhibitory), otherwise $G_{i j}=0$. If $V_{j}$ reaches the threshold $V_{\mathrm{th}}=20 \mathrm{mV}$ at time $t_{n}^{(j)}$, two events are triggered: (i) the membrane potential $V_{j}$ is reset to $V_{r}=10 \mathrm{mV}$ and held fixed for a refractory period $\tau_{r}=0.5 \mathrm{~ms}$; (ii) a spike is emitted and received $\tau_{d}=0.55 \mathrm{~ms}$ later by the postsynaptic cells connected to neuron $j$ according to $G_{i j}$. Except for the system size $N$, all parameters are set as in Ref. [11]: $b=0.8, K=1000$, and $g=5$, so each neuron receives input from $b K[(1-b) K]$ excitatory (inhibitory) presynaptic neurons. Besides this quenched setup, we have considered annealed networks, where the postsynaptic neighbors are randomly chosen at each spike emission. As a matter of fact, in the former (latter) case, the in degree (out degree) is equal to $K$ for each neuron, while the out degree (in degree) is binomially distributed with average $K$ and standard deviation $\sqrt{K}$, in the thermodynamic limit. Our choice was dictated by efficiency of the numerical simulations; however, we have verified that no substantial changes are observed if, instead of fixing the number of links equal to $K$, the probability of each link is set equal to $c=K / N$, as in truly Erdös-Renyi networks.

\section{B. Methods}

A detailed description of network dynamics requires looking both at the microscopic and the macroscopic level.

\section{Microscopic indicators}

The dynamics of a spiking neuron is usually characterized in terms of the probability distribution function (PDF) $Q(T)$ of the ISIs $T$ and of the associated moments: namely, the mean ISI $\bar{T}$ and the standard deviation $\sigma_{T}$ of $T$. Usually, the regularity/irregularity of the dynamics is quantified by the so-called coefficient of variation,

$$
C_{v}=\frac{\sigma_{T}}{\bar{T}},
$$

equal to zero for a periodic dynamics and to 1 for Poissonian spike trains. It should be noted that $C_{v}$ can be larger than 1 for the so-called bursting dynamics, when the neuron alternates 
periods of silence and high activity. The firing rate of a neuron is simply given by $v=1 / \bar{T}$. To characterize the network activity we estimate the mean coefficient of variation $\left\langle C_{v}\right\rangle$ and the mean firing rate $\langle\nu\rangle$, where $\langle\cdot\rangle$ represents an ensemble average over all neurons.

An important observable is the power spectrum $S(f)=$ $\left\langle|\tilde{u}(f)|^{2}\right\rangle$, where $\tilde{u}(f)$ with $f=m /(M \delta t)$ is the Fourier transform of the neural activity $u(t)$, determined by computing the number of spikes emitted in $M$ consecutive time intervals of duration $\delta t$. Further observables we focused on are the phase correlations among different frequencies $f$ and $h$, which can be quantified by the normalized indicator,

$$
D(f)=\sqrt{\frac{\sum_{h \neq f}\left\langle\left|\tilde{u}(h) \tilde{u}^{*}(f)\right|^{2}\right\rangle}{W S(f)}},
$$

where $W=\left\langle\sum_{h}|\tilde{u}(h)|^{2}\right\rangle$ is the total power of the spectrum. One can check that $0 \leqslant D(f) \leqslant 1$, the lower (upper) bound corresponding to uncorrelated (perfectly correlated) channels. The typical values we have used in our simulations are $\delta t=$ $0.11 \mathrm{~ms}$ and $M=2^{15}\left(M=2^{12}\right)$ for the power spectrum (phase correlations) estimation.

\section{Macroscopic indicators}

At the mean-field level, the network evolution is captured by the instantaneous $\operatorname{PDF} P(v, t)$ of the membrane potentials of the neurons. In the limit case of an infinitely large indegree, the perfectly asynchronous regime is characterized by a constant firing rate $\langle v\rangle[20]$. This implies that the flux of neurons along the $v$ axis is independent of both time and potential value, i.e., the corresponding PDF $P_{0}(v)$ should be stationary.

Deviations from stationarity reveal the presence of a collective dynamics. To measure the level of coherence in the network dynamics, a commonly used order parameter is [21]

$$
\rho^{2} \equiv \frac{\overline{\langle v\rangle^{2}}-\overline{\langle v\rangle}^{2}}{\left\langle\overline{v^{2}}-\bar{v}^{2}\right\rangle},
$$

where the overbar denotes a time average. In practice, $\rho$ is the rescaled amplitude of the standard deviation of the average $\langle v\rangle$. When all neurons behave in exactly the same way (perfect synchronization), the numerator and the denominator are equal to one another and $\rho=1$. If instead they are independent as in an asynchronous regime, $\rho \approx 1 / \sqrt{N}$ due to the central limit theorem.

\section{NETWORK DYNAMICS}

\section{A. The quenched network}

Our simulations have mostly been performed by implementing an exact event-driven scheme [22,23]; see Ref. [6] for a description of the details. Since, however, some simulations required implementing a clock-driven Euler scheme and since this latter approach is often used in the literature, we have first compared the two algorithms for a network of $10^{5}$ neurons with a coupling strength $J=0.8$. From the results reported in Fig. 1, we see that the time step $\delta t$ used in the implementation of Euler's algorithm should not be larger than $10^{-3} \mathrm{~ms}$ to get results essentially in agreement with the

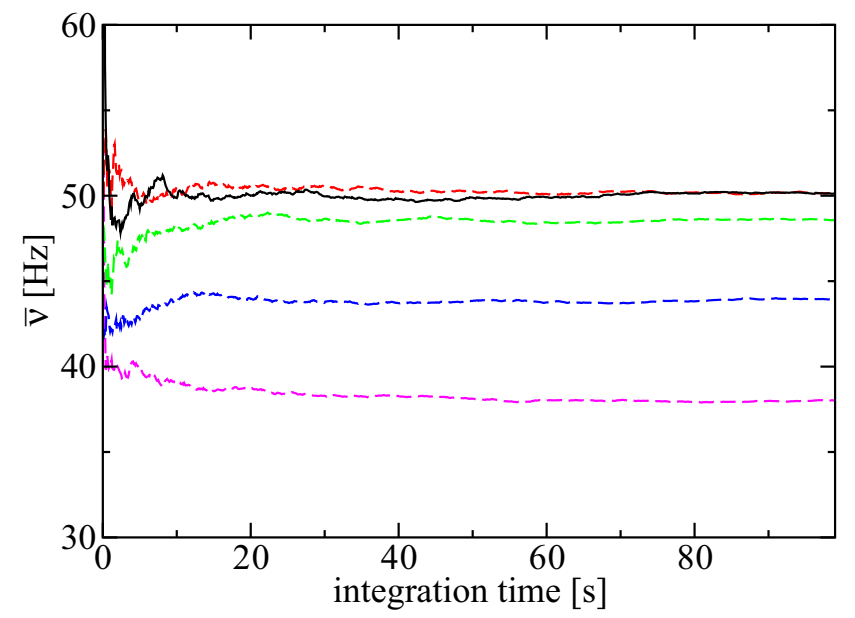

FIG. 1. Running average of the firing rate in a network of $N=10^{5}$ neurons with a coupling strength $J=0.8$ and overall connectivity $K=10^{3}$. The black solid line refers to event-driven simulations; the dashed lines correspond to Euler integration scheme with different time steps $(\delta t=0.1 \mathrm{~ms}, 0.04 \mathrm{~ms}, 0.01 \mathrm{~ms}, 0.001 \mathrm{~ms}$, from bottom to top).

event driven scheme. This is indeed the value employed in our simulations performed with Euler's scheme. Notice that for $\delta t=0.1 \mathrm{~ms}$, a value often chosen in the literature, the firing rate is substantially underestimated (by approximately $24 \%$ ).

We then proceed to analyzing the dependence of the average firing rate $\langle v\rangle$ on the coupling strength $J$. The black, solid curve in Fig. 2(a) has been obtained for $N=10^{4}$ and exactly the same parameter values as in Ref. [11]. As reported therein, $\langle v\rangle$, after an initial drop, increases with the coupling strength $J$. One of the goals of this paper is to understand the origin of this growth in a network where inhibition is expected to prevail over excitation. A theoretical estimate $v_{T}$ of the average firing rate in the asynchronous regime of a sparse network can be derived from the stationary solution of a self-consistent Fokker-Planck equation, under the assumption of an uncorrelated Poissonian activity of the neurons $[3,4]$. However, this prediction, based on the diffusion approximation [3] and reported as a dotted green curve in Fig. 2(a), is able to reproduce only the initial part of the curve $\langle v(J)\rangle$, while it fails to describe the growth observable for larger coupling. Furthermore, in Refs. [5,6] it was found that the corresponding dynamical phase is far from asynchronous; this is testified by the behavior of $\rho(J)$, reported in Fig. 2(b), where we can see that the order parameter $\rho$ can be as large as 0.5 . Considering that the theoretical prediction has been derived under the assumption of a strictly asynchronous regime (i.e., $\rho=0$ ), it is therefore crucial to separate out the effects of the collective dynamics.

This can be done by increasing the network size while leaving the in-degree fixed (namely, $K=1000$ ). Quite surprisingly, the firing rate obtained for $N=10^{5}$ [see the upper blue solid curve in Fig. 2(a)] displays an even more pronounced growth than for $N=10^{4}$, in spite of a weaker synchronization, as shown in Fig. 2(b). The analysis reported in Fig. 2(d), where $\rho$ vs $c=K / N$ is reported for three different synaptic coupling values, shows that the 

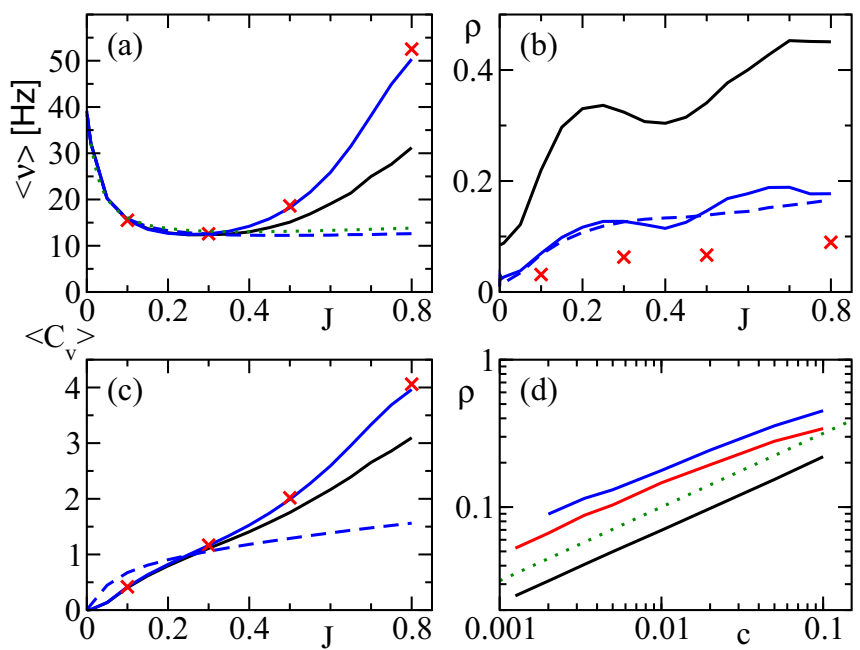

FIG. 2. Collective properties of the network dynamics versus the coupling strength $J$ : (a) average firing rate $\langle v\rangle$, (b) the coherence order parameter $\rho$, (c) the mean coefficient of variation $\left\langle C_{v}\right\rangle$. Solid lines and symbols refer to quenched networks (namely, black lines correspond to $N=10^{4}$, blue ones to $N=10^{5}$, and (red) crosses to $N=8 \times 10^{5}$ ) (blue) dashed lines correspond to simulations of the annealed network performed for $N=10^{5}$ neurons. The (green) dotted line in panel (a) corresponds to the theoretical prediction $v_{T}$ derived in Refs. [3,4]. Finally, panel (d) displays the coherence order parameter $\rho$ versus the connectivity $c=K / N$, reporting only results for the quenched network for different synaptic coupling: namely, $J=0.1, J=0.5$, and $J=0.8$ (solid lines from the bottom to the top). The dotted line refers to the theoretical scaling law $\sqrt{c}$.

collective effects increase as $\rho \approx \sqrt{c}$, consistent with theoretical expectations [4].

Going back to Fig. 2(a), we see that upon further increasing $N$ above $10^{5}$, the firing rate for a given coupling strength $J$ saturates to a finite value. Altogether, we can safely conclude that the increase of $v$ for $J \gtrsim 0.3$ is a genuine property of bona fide asynchronous activity and should be explained as such. Our simulations suggest that the system size $N=10^{5}$ is large enough to ensure nearly asymptotic results and small enough to allow for affordable simulation times. From now on, all simulations will refer to this network size, unless stated otherwise. The main questions we want to address are understanding (i) the features of such a high firing-rate regime and (ii) why it deviates so strongly from the diffusion approximation [3,4], even for a not-too-large synaptic coupling $J$ in a setup where correlations among the different neurons are practically absent.

Before proceeding along these lines, it is useful to provide a more detailed description of the network activity. In Fig. 2(c), the mean coefficient of variation $\left\langle C_{v}\right\rangle$ is plotted versus $J$ for different networks sizes. There, we see that $C_{v}$ steadily increases with $J$ and converges to some asymptotic value upon decreasing $c$. In practice, the neural activity can never be treated as a Poissonian process, as requested by the diffusion approximation employed in Ref. [4]; it is either more regular (for small coupling) or substantially more intermittent (as for $J>0.4$ ). Therefore, it should not come as a surprise
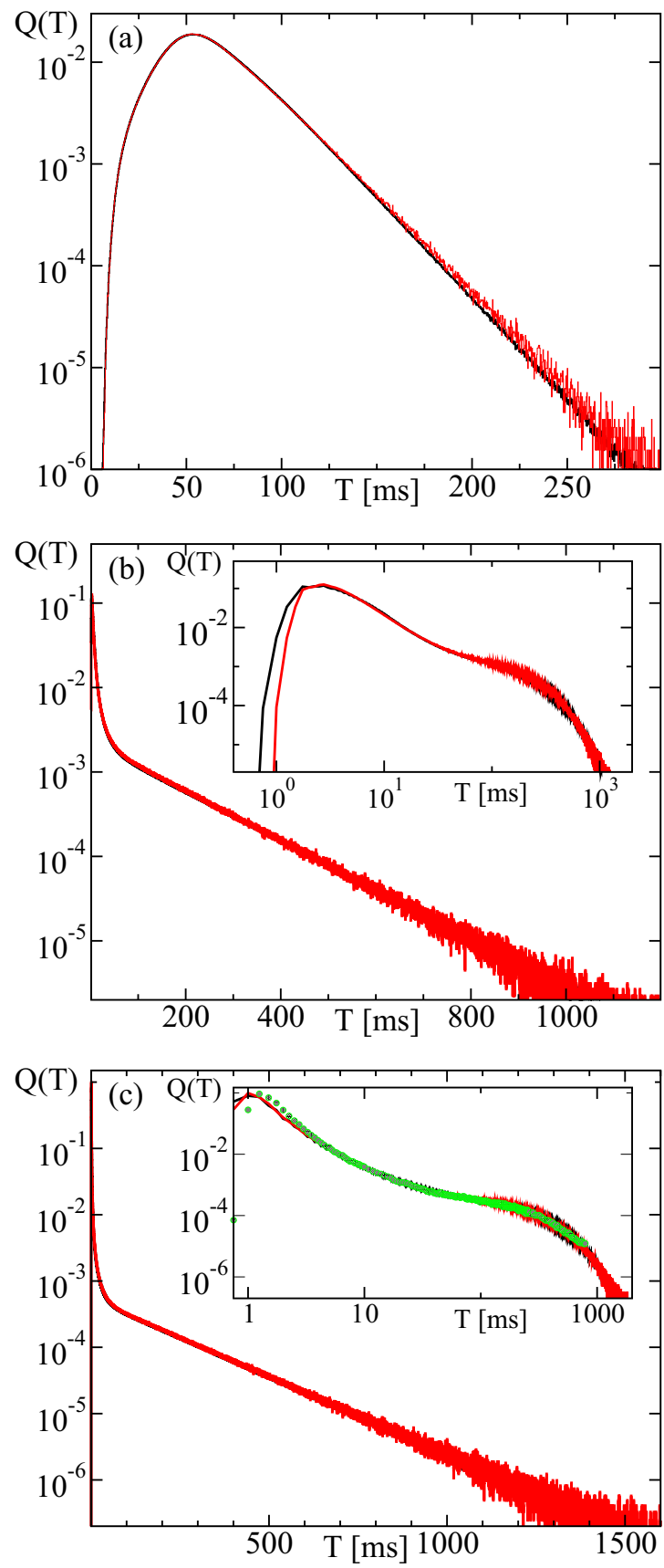

FIG. 3. The PDF $Q(T)$ of the ISIs $T$ generated by the full quenched network (black) and as obtained after the first step of the renewal process recursive procedure (red curve). Panels (a)-(c) refer to $J=0.1, J=0.5$, and $J=0.8$, respectively. The insets contain the same information in log-log scales to emphasize the initial quasipower-law decay. The panel (c) inset shows additionally in green the output of a single neuron subject to a symbol-correlated Poisson process (see Sec. V C).

that a theoretical approach, such as that in Refs. [3,4], based on the assumption that $\left\langle C_{v}\right\rangle=1$, is not accurate.

$C_{v}$ gives only rough information about the distribution of the ISIs. It is worth turning our attention to the full shape of the ISI distribution $Q(T)$. In Fig. 3, we plot $Q(T)$ for $J=0.1$, $J=0.5$, and $J=0.8$; in all cases, we see that for large enough 
ISIs, the PDF is characterized by an exponential tail as for a Poissonian process. However, for small ISIs, the PDF is substantially different. For weak coupling, very small ISIs are strongly inhibited [24]: This is an obvious consequence of the nearly constant input current (mean driven). For stronger couplings, the PDF exhibits a quasi-power-law decay which extends up to 10-20 ms [see the insets of Figs. 3(b) and 3(c)]. These features are consistent with the characterization of the ISI distributions reported in Ref. [25] for spiking neurons driven by fluctuating inputs. In particular, the PDF shown in Fig. 3(a) is expected to emerge when the average effective input current (including the contribution of the synaptic coupling) lies between the threshold and the reset value, which is indeed the case.

Furthermore, we have computed the power spectrum $S(f)$ of single spike sequences. For weak coupling $(J=0.1)$, a small peak is visible at $f=17 \mathrm{~Hz}$ in Fig. 4(a) [26] it is reminiscent of the periodic activity of the uncoupled neurons. At higher frequencies, the spectrum is practically flat, i.e., it is approximately white. Upon increasing the coupling, the spectrum starts exhibiting a low-frequency peak, suggesting the presence of "long"-time correlations. This feature will be further discussed in Sec. V with reference to the emergence of a bursting activity. For $J=0.8$, subsidiary peaks related to the delay emerge for $f=1818 \mathrm{~Hz}$ and its multiples. The delay is always present but for unexplained reasons pops up only for large coupling when the white-noise background is even larger.

\section{B. The annealed network}

So far, we focused on the dynamical properties of a network characterized by a quenched distribution of synaptic connections. However, all theoretical approaches developed to characterize the firing activity do not take into account the actual, invariant structure of the connections. Even more, theoretical approaches do not include delay at all. Therefore, it is natural to ask to what extent the quenched nature of the network may be considered responsible for the observed asynchronous activity. This question can be addressed by considering an annealed network, where the "neighbors" of each given neuron are randomly assigned each time a spike is emitted. More precisely, we proceed as follows: Whenever a neuron fires, we still assume that the quality of the spike (excitatory versus inhibitory) is determined by the neuron itself, but we randomly choose $K$ receiving neurons regardless of their quality. Moreover, we exclude self-connections, i.e., the sender must differ from the receiver. Finally, we keep all parameters as in the quenched network. This guarantees that, on average, each neuron receives $b K$ excitatory inputs and $(1-b) K$ inhibitory ones. Annealed neural networks are usually employed to mimic the effect of unreliable synapses present in the cortex [15,27].

The numerical results for the average firing rate are reported in Fig. 2(a). There we observe a good agreement with the behavior of the quenched network for $J \lesssim 0.3$, while increasing deviations emerge for larger coupling strengths. Interestingly, the behavior of the annealed setup is very close to the theoretical prediction $v_{T}$ [4]. This is not entirely surprising since, as anticipated, the theoretical approach implicitly
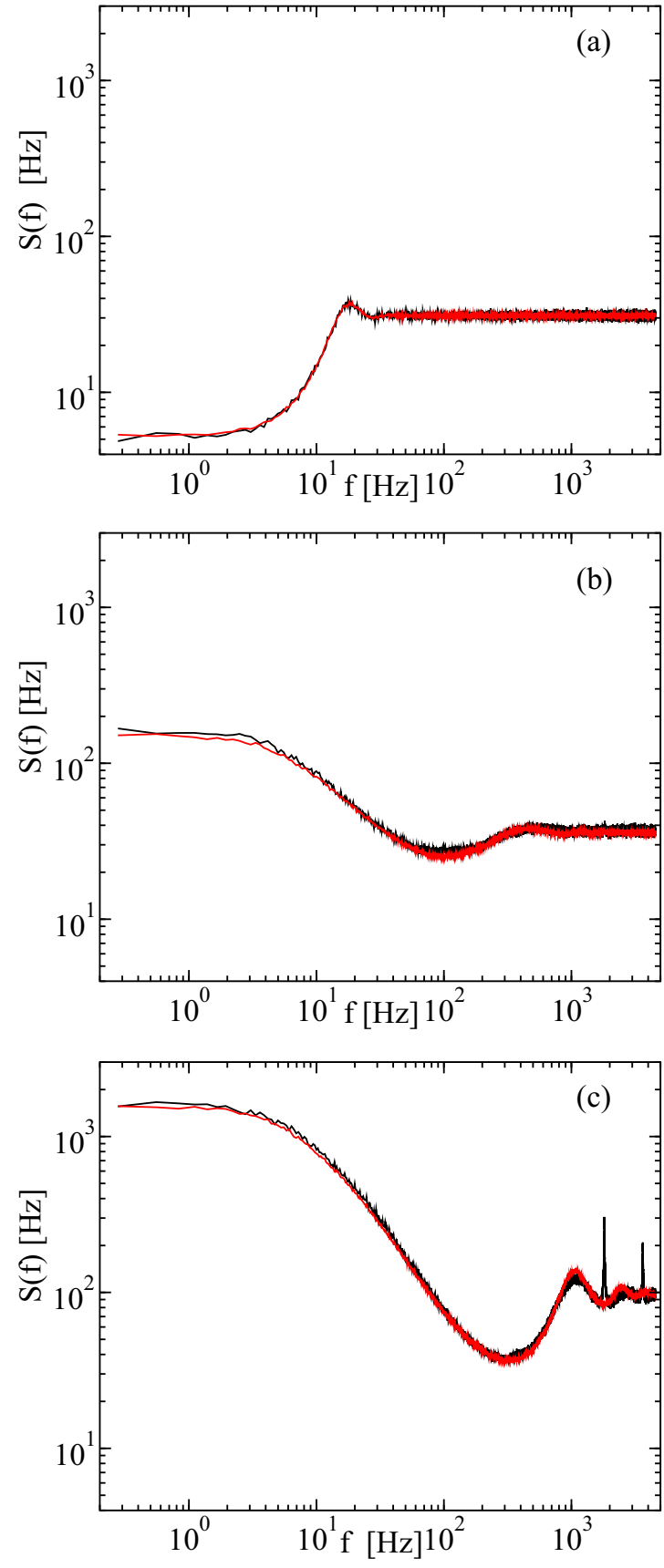

FIG. 4. Power spectra $S(f)$ of the neural activity. Black curves refer to the full quenched network for $N=10^{5}$; red curves refer to the first step of the renewal process approximation. Panels (a)(c) refer to $J=0.1,0.5$, and 0.8 , respectively.

assumes an annealed connectivity. An additional justification for this agreement is the $C_{v}$ values reported in Fig. 2(d) (see the dashed curve) which are much smaller than in the quenched case and closer to 1 , the value corresponding to a Poisson process.

\section{SELF-CONSISTENCY}

In the previous section, we have seen that quenched and annealed networks behave in a substantially different way, when the coupling strength is larger than $J=0.25$. To what 
extent is this difference the signature of the crucial role played by a fixed structure of the synaptic connections?

In this section, we address this issue by implementing a self-consistent approach, where the input current is assumed to be the superposition of independent signals, each sharing the same "statistical" properties of the single-neuron activity. Two different approximation schemes are hereby discussed: (i) the hypothesis of a perfect RP and (ii) mutually uncorrelated frequency channels (also termed Gaussian approximation). Below we show that the former one provides a more accurate representation of the neural activity.

\section{A. Renewal process}

A RP is fully characterized by the ISI probability distribution $Q(T)$. Assuming $Q(T)$ is known, a typical spike sequence can be readily generated by randomly drawing a series of $T$ values according to this distribution. At variance with Ref. [9], where the authors suggested the idea of approximating the synaptic current with a RP, here we limit ourselves to assume that the single-neuron output activity is a RP, but we do not extend the assumption to the input, which is treated as the superposition of $K$ independent RPs. This is an important difference since, as already remarked in Ref. [28], the superposition of RPs is not renewal itself unless the single processes are purely Poissonian (this is not our case). So, at variance with Ref. [9], we relax the condition of a strictly renewal input process and replace this ansatz with the more general hypothesis of a superposition of independent RPs.

In practice, we have implemented the following recursive procedure: given the ISI distribution $Q_{k}(T)$ determined in the $k$ th step, we have generated the synaptic current $R I$ of a generic neuron [in the $(k+1)$ st step] by superposing $K$ independent RPs all built according to the same distribution $Q_{k}(T)$ (under the constraint that $b K$ spikes are excitatory and the remaining ones inhibitory). Upon afterward integrating the single-neuron equation, we have generated the firing activity induced by the current $R I$, thereby determining the $(k+1)$ st distribution $Q_{k+1}(T)$.

We first focused on $J=0.8$, since the theoretical prediction $v_{T}$ [3] is significantly inaccurate for this coupling strength. The initial condition $Q_{0}(T)$ has been selected as the distribution generated by a quenched network of $N=$ $10^{5}$ neurons with an in-degree $K=1000$. The corresponding firing rate is $\langle v\rangle=50.4 \mathrm{~Hz}$ and its coefficient of variation is $\left\langle C_{v}\right\rangle=3.97$ [29]. This pair of values is represented by the point $P_{q}$ in Fig. 5: It corresponds to the projection of the asynchronous state of the quenched network in this twodimensional space. The iterates of the recursive procedure have been projected on the same plane; they are so close to each other to be hardly discernible in the main panel (see the enlarged plot presented in the upper inset of Fig. 5 for a clearer representation). The closeness among consecutive iterates is confirmed by the shape of the ISI distribution: In Fig. 3(c), we see that $Q_{1}(T)$ is practically indistinguishable from $Q_{0}(T)$. Altogether, these observations strongly hint at the existence of a fixed point of the RP recursive procedure in the vicinity of $P_{q}$. Further iterates start separating from each other, suggesting that the fixed point is a saddle, which initially attracts the trajectory along the stable manifold and

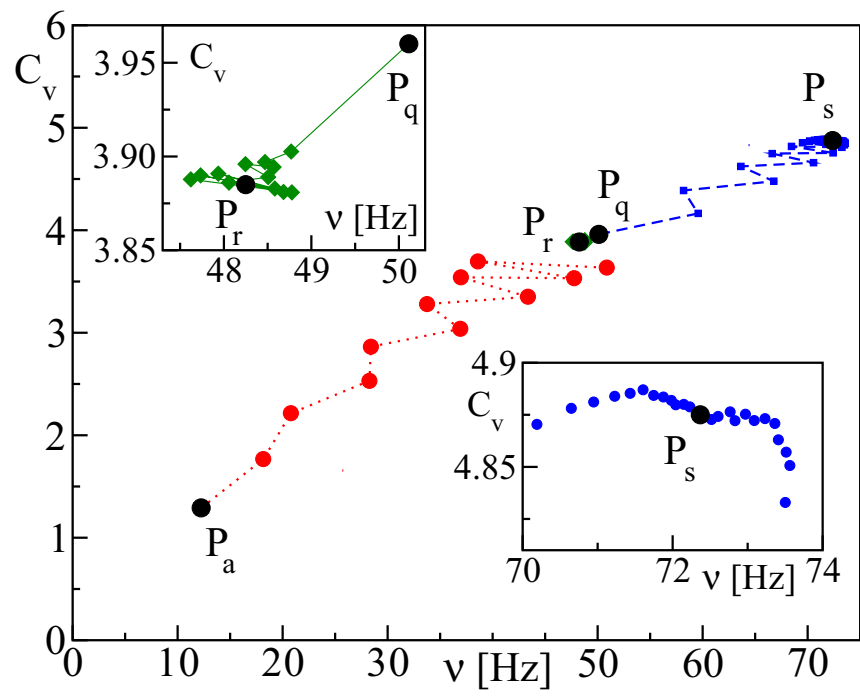

FIG. 5. Results of the recursive procedure based on the renewal process and the Gaussian approximation. $P_{q}$ and $P_{a}$ identify the dynamics of quenched and annealed networks, respectively. Red circles (green diamonds) refer to the iterates of the RP iterative procedure while starting from $P_{a}\left(P_{q}\right)$; green diamonds are better visible in the enlargement reported in the upper inset, where $P_{r}$ denotes the fixed point of the renewal process approximation. Blue squares refer to the iterative process based on the Gaussian approximation: $P_{q}$ is the initial condition, while $P_{s}$ denotes the fixed point of this approach (see the lower inset for an enlargement of the later stages). All data have been obtained for $J=0.8$ ).

eventually drives it away along the unstable manifold. If, in analogy to what was done in Ref. [10] for the Gaussian approximation, we include memory effects by building the new ISI PDF as the average of the last two distributions, the saddle is stabilized: The resulting fixed point is represented in Fig. 5 as $P_{r}$. The nonperfect correspondence between $P_{q}$ and $P_{r}$ may have a double rationale: the RP assumption is not exact; the network size used to determine $P_{q}$ is not large enough.

To test the quality of the RP approximation, we have studied the correlations of the sequence $T_{n}$ of consecutive ISIs by estimating the so-called serial correlation coefficient [30]:

$$
C(m)=\frac{\left\langle T_{n+m} T_{n}\right\rangle-\left\langle T_{n}\right\rangle^{2}}{\left\langle T_{n}^{2}\right\rangle-\left\langle T_{n}\right\rangle^{2}} .
$$

In a strictly RP, $C(m)=0$ for $m \geqslant 1$. Tests made on the neurons of a quenched network for $N=10^{5}$ show that $C(1)$ is at most of order $10^{-3}$, suggesting that the neural activity is well approximated by an RP. On the other hand, since the order parameter $\rho$ is still relatively large for $N=10^{5}$ $(\rho=0.17)$, finite-size effects are probably the predominant source of differences between $P_{q}$ and $P_{r}$.

What if the same recursive procedure is applied, starting from the dynamical regime exhibited by the annealed network (see point $P_{a}$ in Fig. 5). Forward iterates rapidly move away from $P_{a}$ and approach $P_{r}$ (see the full circles in Fig. 5). The increasing amplitude of the "transversal" fluctuations confirm that $P_{r}$ is a saddle point. Furthermore, the recursive procedure shows that $P_{a}-$ a fixed point of the annealed process-is not 


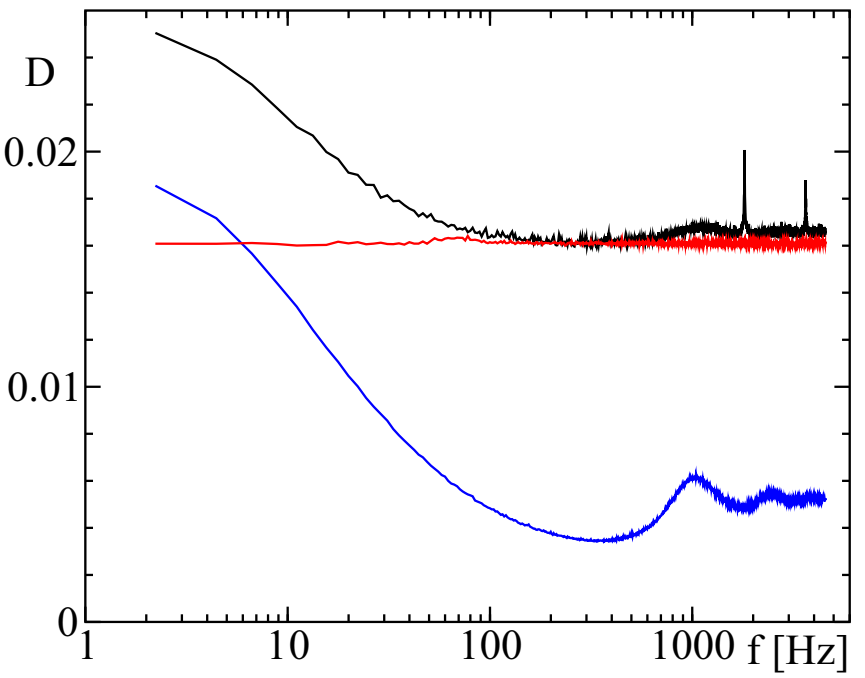

FIG. 6. Degree of phase correlations $D(f)$ of the Fourier transform of the spiking activity for $J=0.8$. The lowermost blue line corresponds to the RP; the uppermost black solid line corresponds to the activity of the quenched network. Finally, the red line corresponds to the annealed network.

a fixed point of the RP iterative procedure. The reason is that while the temporal correlations exhibited by the single-neuron activity (encoded in the bursting activity) are preserved by the RP approximation, they are lost in the annealed setup because of the random reshuffling of the synaptic connections. The separation between $P_{a}$ and $P_{r}$ implicitly suggests the important role played by the bursting activity as will be confirmed in the following section.

Finally, we have also implemented the RP approach for smaller $J$ values, always finding evidence of a weakly unstable fixed point (actually, the degree of instability decreases upon decreasing $J$ ). The resulting message is that the stable asynchronous dynamics exhibited by the quenched network is well reproduced by an unstable fixed point of a recursive transformation based on the RP approximation.

\section{B. Power spectrum}

Fourier analysis offers the opportunity for additional verification of the validity of the RP approximation. In Fig. 4, we compare the power spectrum of the single-neuron activity after the first iterate obtained under the RP approximation [31] (see the purple curve) with the spectrum exhibited by the quenched network. The agreement is quite good for all three tested coupling strengths, the major discrepancy being the absence of peaks at multiples of $v_{d}=1818 \mathrm{~Hz}$ for $J=0.8$, which cannot be reproduced by the RP approximation, since the delay is not included in such formulation.

Next, we have directly estimated the correlations among the phases of the Fourier modes by computing $D(f)$ [see Eq. (3)] both for the quenched network and the RP approximation (see upper and lower curves in Fig. 6, respectively). Phase correlations appear to be small in both cases (look at the vertical scale): We attribute the larger amplitude exhibited by the quenched network to the presence of a residual collective dynamics, absent by definition in the RP approximation. A

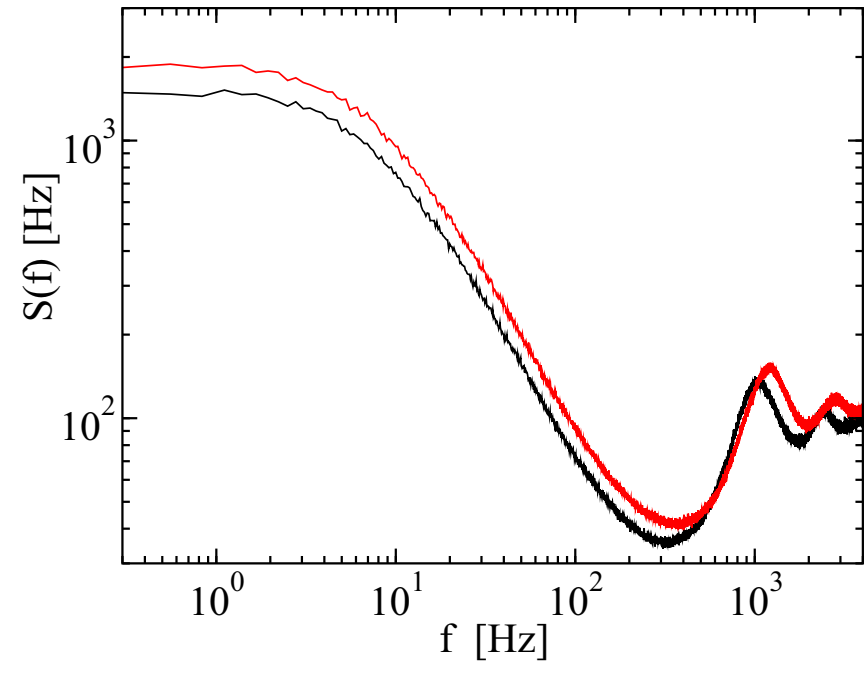

FIG. 7. Power spectra for the network activity. The black curve corresponds to the spectrum of the activity of a single LIF neuron driven within the RP approach (it is basically indistinguishable from the true quenched network activity). The red curve is obtained by randomizing the phases of the input signal. Data refers to $J=0.8$.

comparably small level of correlations is also found in the annealed network (see the almost flat red line).

As a second test of the relevance of phase correlations, we have investigated the consequence of phase randomization within the RP procedure. More precisely, given the synaptic current $u(t)$ and its Fourier transform $\tilde{u}(f)$, we have generated a new transform $\tilde{u}_{N}(f)=|\tilde{u}(f)| e^{i \phi(f)}$ by randomly assigning the phase $\phi(f)$ to the frequency $f$. A new signal $u_{N}(t)$ is then obtained by back transforming $\tilde{u}_{N}(f)$. The resulting spectrum of the firing activity of a neuron subject to the current $u_{N}(t)$ is presented in Fig. 7 (see the red curve). The difference with the original spectrum (see the lower black curve) is not entirely negligible: It is around 20\% in the low frequency region.

Finally, we have implemented the recursive procedure proposed in Ref. [9], here briefly recalled. Let $S_{k}^{(o)}(f)$ denote the power spectrum of the single-neuron spiking activity at the $k$ th recursive step. Also, let $S_{k+1}^{(i)}(f)$ denote the power spectrum of the synaptic current in the $(k+1)$ st recursive step. In the asynchronous regime, the synaptic current is the superposition of $K$ independent signals $[b K$ excitatory and $(1-b) K$ inhibitory], all characterized by the same spectrum $S_{k}^{(o)}(f)$. Taking into account the amplitude of the single spikes, we have that $S_{k+1}^{(i)}=\left[J^{2} K(b+(1-b) g)\right] S_{k}^{(o)}(f)$ [32]. The definition of the procedure is completed by adding the "rule" to generate $S_{k+1}^{(o)}$, given $S_{k+1}^{(i)}(f)$. This is done by feeding a single neuron with a phase-randomized spectrum (see the paragraph above). The self-consistent solution is finally identified by the condition $S_{k+1}^{(o)}=S_{k}^{(o)}$.

We have implemented this approach with an Euler integration step $\delta t=10^{-3} \mathrm{~ms}$ starting from the initial condition $P_{q}$, the best proxy for the asynchronous regime. The first 33 iterates are reported in Fig. 5, where we see that they move away from $P_{r}$ (see the blue squares) and approach a seemingly stable fixed point $P_{s}$. The relatively large difference between $P_{s}$ and $P_{q}$ suggests that this approximation scheme is not as accurate as the RP method and implicitly means that the phase 


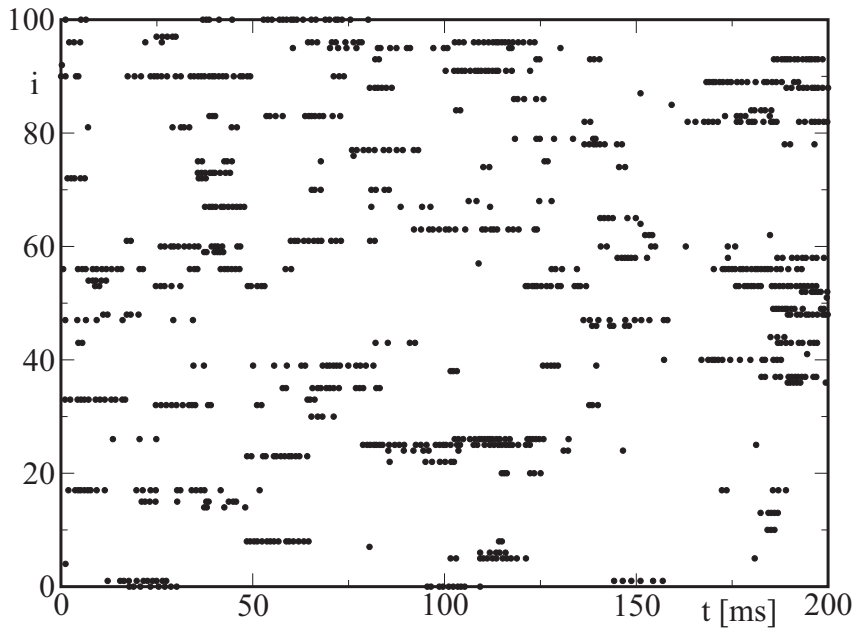

FIG. 8. Raster plot of the firing activity in the quenched network with $N=100000$ and coupling $J=0.8$. To illustrate the busting activity of single neurons, we display the spiking activity of only 100 neurons over a short time interval.

correlations built while integrating the LIF equation are not negligible [33].

\section{BURSTING ACTIVITY}

In the previous sections, we have seen that for strong coupling the neural activity is characterized by a large $C_{v}>1$, a typical indication of bursting activity. This is indeed qualitatively confirmed in Fig. 8, where a raster plot is reported of the activity of a few neurons: Irregular bursts of spikes intermingled by long period of silence are clearly visible. Some neurons seem to be completely silent but this is just the consequence of the finite length of the time window (all neurons fire with the same rate). In this section, we discuss in more quantitative terms this form of asynchronous dynamics, starting from the basic question of how it is possible for it to be self-sustained.

In the asynchronous regime, the average input current induced by the synaptic coupling is

$$
\langle I\rangle=\frac{J}{R}\left(n_{E}-g n_{I}\right)=\frac{K J}{R} v[b(g+1)-g],
$$

where $n_{E}\left(n_{I}\right)$ denotes the number of excitatory (inhibitory) spikes received per time unit. Depending on whether $R_{0}\langle I\rangle$ is larger or smaller than $V_{\text {th }}-R I_{0}=-4$, the neuron operates either above or below threshold. In fact, in the latter case, the velocity field crosses the zero axis below the threshold $V_{\text {th }}$, preventing threshold passing. In Fig. 9, we plot the firing rate versus $\langle I\rangle$ for different values of the coupling strength $J$ (see the solid line): increasing $J$ corresponds to moving leftward along the curve, starting from the rightmost point, which corresponds to the uncoupled limit. Upon increasing $J$, $\langle I\rangle$ decreases monotonically: This is the consequence of the prevalent inhibition. At the same time, the firing rate, after an initial drop, starts growing; this happens for $J \approx 0.25$, as it can be inferred by comparing with Fig. 2(a). The increase continues also when the neuron operates below threshold and surpasses the activity of the uncoupled regime.

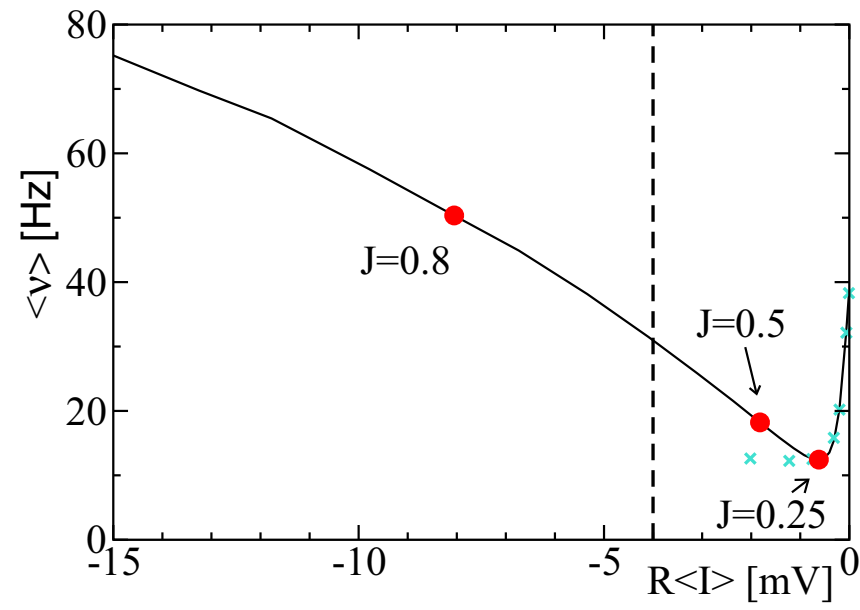

FIG. 9. Firing rate $\langle v\rangle$ versus the synaptic "current" $R\langle I\rangle$ for different coupling strengths for the quenched network (solid line and red circles). Zero coupling corresponds to the rightmost point, where the synaptic current obviously vanishes. The vertical dashed line separates the region where neurons operate above (right) from below (left) threshold. The green crosses report the outcome of the annealed network. The two crosses deviating from the solid line belong to $J=0.5$ and 0.8 in the annealed setup.

In the same figure, we also report the outcome of annealednetwork dynamics (see the crosses): for small $R\langle I\rangle$, i.e., for small coupling we see an almost perfect coincidence. On the other hand, by further decreasing the internal current (i.e., upon increasing the coupling strength), the firing rate does not increase in the annealed network, confirming the qualitatively different behavior exhibited by the two setups. The annealed network operates above threshold.

\section{A. Correlations between membrane potential and synaptic current}

The counterintuitive activity displayed by the quenched network requires an explanation. We have verified that the effective self-induced excitation is not the result of a symmetry breaking: All neurons (both excitatory and inhibitory) behave in the same way, as they should. More instructive information can be extracted by exploring the correlations between the actual value of the membrane potential and the quality (excitatory versus inhibitory) of the spike received by a given neuron. In other words, we have computed the relative fraction $s_{E}(V) d V$ of excitatory spikes received when $V \in$ $[V, V+d V]$. If the receiving times were uncorrelated with the membrane potential, then $s_{E}(V)$ would be independent of $V$ and equal to $b$. Actually, this is expected within the framework of a $\delta$-correlated input signal as assumed in Ref. [4].

Instead, in Fig. 10(a), we see sizable deviations, especially in the vicinity of $V_{\mathrm{th}}$, where $s_{E}$ is significantly larger than $b$, hinting at a higher excitation than a priori foreseeable. We have verified that, as expected, the average of $s_{E}$-computed along the $V$ axis and weighted according to the stationary distribution $P(V)$ - is equal to $b$; see the horizontal line.

A perhaps more enlightening representation of the role played by the $V$ dependence of $s_{E}$ is proposed in Fig. 10(b), 

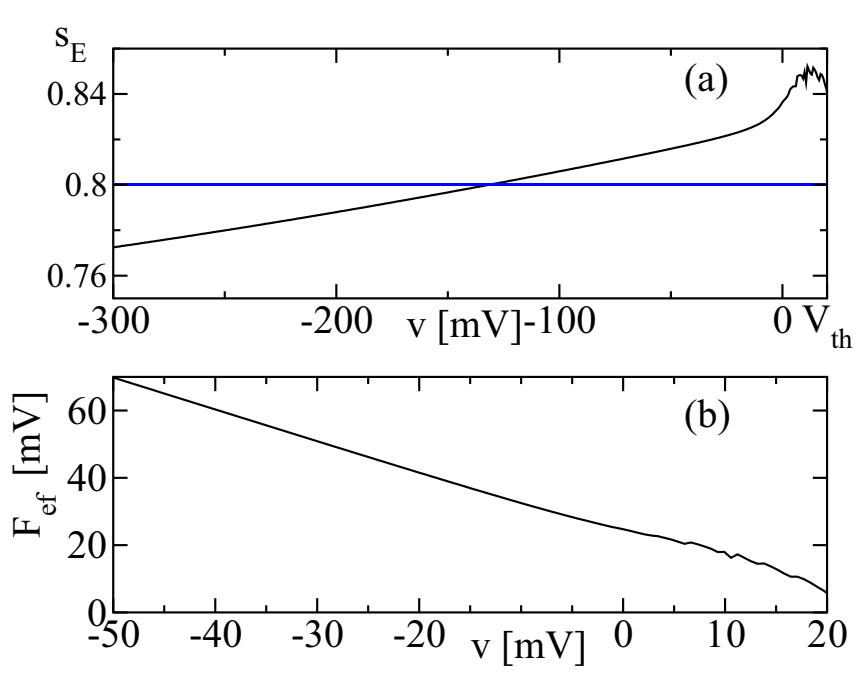

FIG. 10. (a) Fraction $s_{E}$ of excitatory spikes versus the current value $V$ for $J=0.8$. The horizontal line corresponds to the average global fraction $b=0.8$. (b) Effective velocity field $F_{\text {eff }}$ as from Eq. (6).

where we plot the effective velocity field

$$
F_{\text {eff }}=R I_{0}-V+K J \tau\langle v\rangle\left[s_{E}(1+g)-g\right],
$$

where the $V$-dependent $s_{E}$ replaces $b$. Interestingly, the effective velocity field does not cross the zero axis below threshold, showing that the neuron effectively operates above threshold, in spite of $R\langle I\rangle<V_{\text {th }}-R I_{0}$. So, we can conclude that including the $V$ dependence of $s_{E}$ into the neural dynamics helps solve the paradox of a neuron operating on average below threshold. On a more quantitative level, we can interpret Eq. (6) as a deterministic evolution equation and thereby compute the firing rate $v_{a}$ as the inverse of the time needed to reach the threshold $V_{\text {th }}$, while starting from $V_{r}$ (augmented by the refractory time). By inserting $v$ and $s_{E}(V)$ values obtained from the network simulations for $J=0.8$, we find $v_{a}=77 \mathrm{~Hz}$, to be compared with the observed rate $v=50 \mathrm{~Hz}$. The agreement is not as good as one might have hoped for, but it should also be noted that Eq. (6) does not account for the (strong) input fluctuations!

Moreover, the $V$ dependence of $s_{E}$ still needs to be understood. Some light can be shed by arguing as follows. Let us introduce the joint probability $P(E, L)$ that an excitatory spike reaches the neuron, when its membrane potential $V \in$ $L=\left[V_{0}, V_{\text {th }}\right]$, where $V_{0}$ is selected as the point where $s_{E}=b$, The standard Bayesian inference rule implies that

$$
P(E \mid L)=\frac{P(L \mid E)}{P(L)} P(E),
$$

where $P(A \mid B)$ denotes the probability of observing $A$, given $B$; moreover $P(E)=b$, while $P(L)$ is the probability of $V>V_{0}$, and $P(E \mid L)$ is just the average of $s_{E}$ over $L$. Let us now focus on $P(L \mid E) / P(L)$ : This is the probability of $V>V_{0}$ when an excitatory spike arrives (rescaled to the unconditional probability to stay in $L$ ). If excitatory spikes arrive in bursts, for many of them the corresponding $V$ value is relatively large as a consequence of the excitation provided by the previous spikes. Therefore, it is natural to expect $P(L \mid E) / P(L)>1$.
This is precisely what we see in Fig. 10, where one can notice that $s_{E}$ is larger than $b$ close to threshold. Consistency then imposes that $s_{E}<b$ further away.

\section{B. Synaptic current: An Ornstein-Uhlenbeck process}

The role of correlations can be analyzed from a different point of view: Since the neuron is typically under the action of a negative current, its membrane potential is kept away from threshold $\left(V<V_{\text {th }}\right)$. Only when relatively strong positive fluctuations of the input current arise, can the neuron overcome the threshold and emit a spike. If the correlations are sufficiently long lasting, the fluctuation may stand long enough to allow for the emission of a sequence of spikes and give rise to a "burst." This mechanism has already been investigated in the past to quantify the spiking activity of a subthreshold neuron subject to Ornstein-Uhlenbeck (OU) noise, finding that a long correlation time gives rise to a bursting activity [30,34]. Unfortunately, we cannot make use of their formulas, since the correlation time is not much longer than $\tau$. We can, nevertheless, proceed in a purely numerical way by approximating the input current $I$ with the outcome of an OU stochastic equation, namely,

$$
\tau_{c} \dot{I}=\langle I\rangle-I+\xi,
$$

where $\langle I\rangle$ is the average current observed in the numerical simulations of the quenched network, while $\tau_{c}$ is the input correlation time and, finally, $\xi$ is a $\delta$-correlated white noise $\left\langle\xi\left(t^{\prime}\right) \xi\left(t^{\prime}+t\right)\right\rangle=\sigma^{2} \delta(t)$. We have thereby tuned $\tau_{c}$ and $\sigma^{2}$ until the neuron activity is characterized by the expected firing rate and the corresponding $C_{v}$. For $J=0.8$, we have found $\tau_{c} \approx 160 \mathrm{~ms}$ and $\sigma^{2} \approx 0.062$. As a bonus, the resulting ISI distribution turns out to be quite similar to the expected one, the major difference being the peak which, instead of being located in $T=1 \mathrm{~ms}$ [see the inset in Fig. 3(c)], occurs for $T \approx 4 \mathrm{~ms}$. Altogether, one can nevertheless conclude that the OU approximation provides a reasonable description of the input current.

We have implemented the same procedure for $J=0.5$ : In spite of the similar bursting activity, the neuron operates on average above threshold and we have not found any way to parametrize the OU process so as to reproduce the observed activity. On the other hand, a good reproduction of the neural activity is found for $J=1$ by assuming $\tau_{c}=145$ and $\sigma^{2}=0.28$. Two interesting comments are in order: (i) upon increasing the coupling, the correlation time does not increase-it seems that $\tau_{C} \approx 140-160$ is an intrinsic property of the network, and (ii) the noise amplitude increases by more than a factor of 4 , while passing from $J=0.8$ to $J=1$ and this is the reason why the firing rate is larger in the latter case, even though the neuron operates much more below threshold. The increase of the effective noise can be attributed to two factors: A minor contribution comes from the increased coupling strength (from 0.8 to 1 ); a more relevant contribution is the increased fluctuations of the single-neuron activity quantified by the $C_{v}$. 


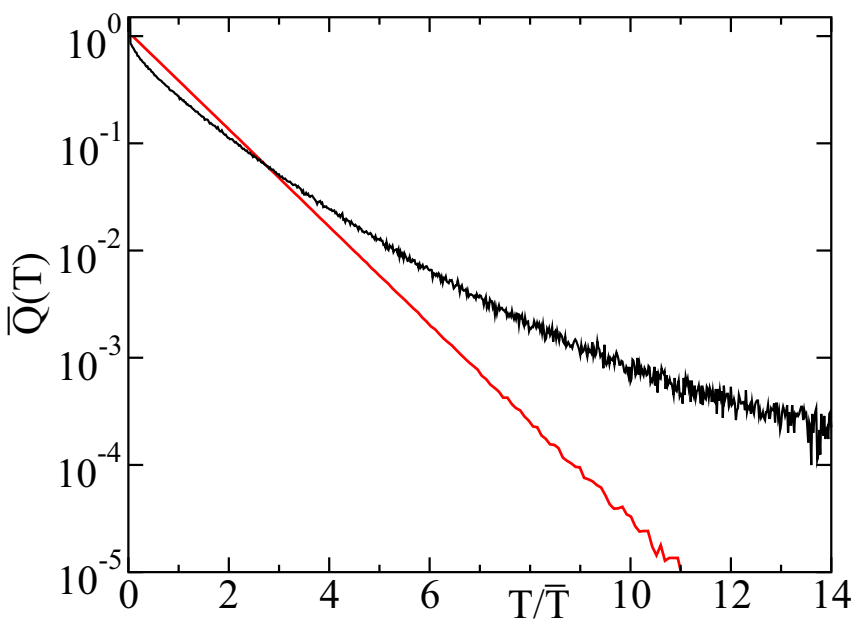

FIG. 11. The effective distribution $\bar{Q}(T)$ of interexcitatory spikes received by a single neuron for $J=0.8$. The black curve refers to the quenched network, the red one to the renewal process approximation. The horizontal axis is rescaled by the mean firing rate of the respective population of excitatory spikes.

\section{Synaptic current: Symbolic correlations}

We conclude this section by looking at correlations from a different point of view. As shown in Sec. IV, the output activity of the single neuron is well approximated by a RP, but we do not expect the same to be true for the input, obtained by superposing $K$ independent such processes. To investigate the way correlations manifest themselves, we separately computed the ISI distribution of all excitatory and inhibitory spikes received by a given neuron.

In Fig. 11, we report the ISI distribution of excitatory spikes (inhibitory spikes follow the same statistics) for both the original quenched network (black curve) and within the RP approximation (red curve). $\bar{T}$ represents the average separation between consecutive spikes, i.e., $\bar{T}$ is equal to the average single-neuron ISI divided by 800 - the total number of incoming excitatory synaptic connections. The red curve follows a clean Poissonian distribution, while the quenched network exhibits a slower than exponential decay (in this time range); furthermore, in the latter case, the first channel is very large because of the unavoidable presence of avalanches occurring in the quenched setup (see Ref. [6]). We attribute most of the deviations from a pure exponential to the residual presence of collective dynamics. In any case, this discrepancy is a minor issue: The relevant correlations are those between excitatory and inhibitory spikes, as revealed by the following test. We have fed a single neuron with two different signals: (i) a perfectly Poisson process composed of independent excitatory and inhibitory spikes and (ii) a synthetic signal built by assuming a Poisson distribution of consecutive spikes with the same rate as the quenched network, but keeping the original symbolic ordering (i.e., EEIEEEIE..., where the letters $E / I$ means that the spike is either excitatory or inhibitory) observed in the quenched network.

The resulting membrane-potential distributions of the neuron are reported in Fig. 12. The black curve, obtained by using the above-mentioned synthetic signal, is very similar to

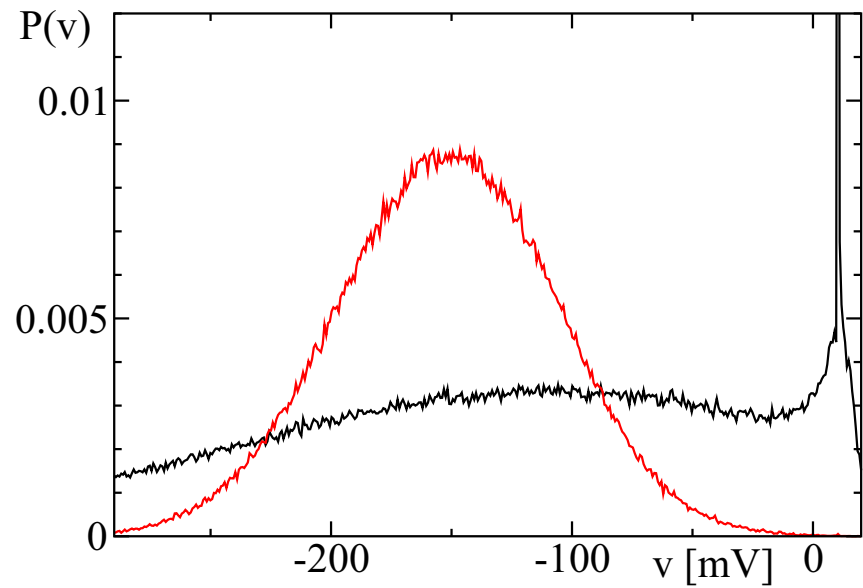

FIG. 12. Probability density of the membrane potential $P(v)$. The black curve exhibiting the divergence in $v=10$ corresponds to the synthetic signal described in text; the red curve is obtained by feeding the neuron with a purely Poisson process with the same firing rate.

the original distribution. On a quantitative level, the resulting firing rate, the $C_{v}$, and the PDF of the ISIs are very close to the values exhibited by the RP approximation (deviations are smaller than 1\%): see the inset of Fig. 3(c). On the other hand, the red curve, originating from the strictly Poisson process is shifted toward very negative $v$ values and nearly vanishes close to the threshold, suggesting a very low firing activity as indeed observed.

Altogether, this proves that the relevant correlations are contained in the symbolic representation of the spike sequence. The very negative values of the membrane potential displayed in Fig. 12 can raise doubts about the validity of the LIF model. However, it should be recalled that the exponential integrate and fire neuron [35], successfully employed to reproduce the neurophysiological characteristics of pyramidal neurons in the cortex [36], reduces to the LIF neuron for very negative membrane potentials. Moreover, the strong coupling strength selected in this paper has been chosen to highlight the role of correlations, but the phenomenon persists at lower coupling strengths too.

\section{CONCLUSIONS AND OPEN PROBLEMS}

In this paper, we have shown that upon increasing the coupling strength $J$ (and for $J>0.25$ ), a slightly inhibitory sparse network of LIF neurons operates increasingly below threshold and yet fires at an increasingly fast rate. This claim is supported by careful numerical simulations, tailored so as to marginalize the effects of collective synchronization.

This counterintuitive, self-sustained activity observed in quenched networks, disappears in annealed networks, i.e., in setups where the synaptic connections are continuously randomly reshuffled. In the latter case, the neural activity is both weaker and more homogeneous (for $J=0.8$, the firing rate drops by a factor of about 4 ). The difference between quenched and annealed setups is reminiscent of replica symmetry breaking [16], but the anomaly of the phenomenon is mitigated by the observation that the quenched-network 

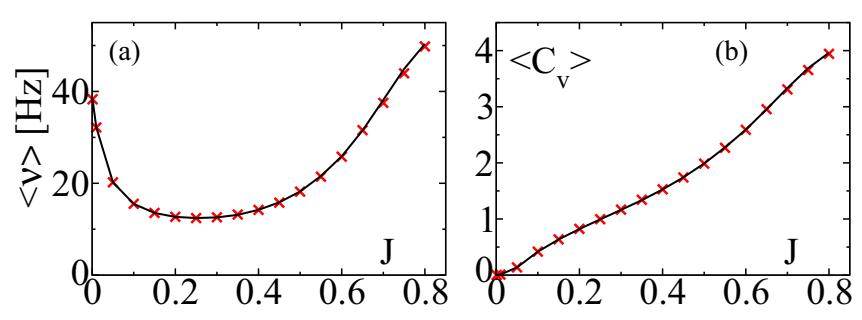

FIG. 13. Neural activity for different delay times. The solid curves all refer to $\tau_{d}=0.55$, while crosses refer to $\tau_{d}=0.3$. All simulations are performed in a network with $N=10^{5}$ neurons and a connectivity $K=1000$.

dynamics can be reproduced to a high degree of accuracy by an approach, the RP approximation, which does not take into account the structure of the synaptic connections. Still, the comparison between quenched and annealed dynamics (see Fig. 9 for the most enlightening representation) seems to suggest the presence of a phase transition when $J$ is increased. It looks like the two regimes deviate from one another above $J \approx 0.25$. This is reminiscent of the claim made by Ostojic about the existence of two distinct asynchronous regimes [11]. This claim has been criticised in Ref. [17]; we are also unable to find evidence of a qualitative difference between the two regimes (above and below a supposedly critical point $J_{c}$ ).

Within the RP approximation, the neural activity is fully characterized by the ISI distribution. In the limit of large coupling strengths, such a distribution exhibits a power-law decay, similar to what was found while studying the response of a single neuron to OU processes $[30,34]$ and similar to experimental observations made in the sensorimotor cortex of rats performing behavioral tasks [37]. It should, however, be noticed that in our case, the scaling range is much smaller than in the experimental observations.

The recursive process based on the RP approximation proves rather accurate in spite of not taking into account the delay. It is therefore natural to ask whether this is also true in the quenched network. Simulations performed for different delay values confirm a substantial independence of the outcome on $\tau_{d}$. In Fig. 13, we compare the firing rate and the $C_{v}$ obtained for $\tau_{d}=0.3$ with the original simulations (performed for $\tau_{d}=0.55$ ).

Last but not least, we wish to comment on the peculiar behavior of the network observed for large coupling strengths. The strong firing activity is self-sustained by its burstiness (signaled by the large $C_{v}$ values), which, de facto, provides the relatively long correlations required to let a neuron below threshold fire. This clarifies the reason why the theoretical formula based on the assumption of $\delta$-correlated current fluctuations fails to reproduce this regime. Recently, a more sophisticated self-consistent approach has been developed, where the Fokker-Planck equation has been augmented to account for temporal correlations in the synaptic current [38]. Its (numerical) implementation to a weak-bursting regime looks promising. It will be worth exploring its validity in a more inhibition-dominated regime such as the one explored in this paper. Interestingly, the bursting activity is reproduced also assuming a strictly Poisson ISI distribution, but retaining the correlations contained in the symbolic representation of the spike types (i.e., excitatory versus inhibitory). A simple quantification of such correlations might open yet another route for a quantitative characterization of the neural activity.

Finally, we wish to stress that no specific properties of the LIF neurons have been invoked for our approach to be valid. Accordingly, we expect the RP approximation to be applicable to different neural models such as the exponential and the quadratic integrate and fire neurons, or conductance-based models.

\section{ACKNOWLEDGMENTS}

A.T. received financial support by the Excellence Initiative I-Site Paris Seine (Grant No. ANR-16-IDEX-008), by the Labex MME-DII (Grant No ANR-11-LBX-0023-01) (together with A.P. and E.U.), and by the ANR Project ERMUNDY (Grant No ANR-18-CE37-0014), all part of the French program Investissements d'Avenir.
[1] A. S. Ecker, P. Berens, G. A. Keliris, M. Bethge, N. K. Logothetis, and A. S. Tolias, Science 327, 584 (2010).

[2] A. Renart, J. de la Rocha, P. Bartho, L. Hollender, N. Parga, A. Reyes, and K. D. Harris, Science 327, 587 (2010).

[3] R. M. Capocelli and L. M. Ricciardi, Kybernetik 8, 214 (1971).

[4] N. Brunel, J. Comput. Neurosci. 8, 183 (2000).

[5] E. Ullner, A. Politi, and A. Torcini, Chaos 28, 081106 (2018),

[6] A. Politi, E. Ullner, and A. Torcini, Eur. Phys. J. Spec. Top. 227, 1185 (2018).

[7] M. J. E. Richardson and R. Swarbrick, Phys. Rev. Lett. 105, 178102 (2010).

[8] S. Olmi, D. Angulo-Garcia, A. Imparato, and A. Torcini, Sci. Rep. 7, 1577 (2017).

[9] B. Dummer, S. Wieland, and B. Lindner, Frontiers Comput. Neurosci. 8, 104 (2014).

[10] R. F. O. Pena, S. Vellmer, D. Bernardi, A. C. Roque, and B. Lindner, Frontiers Comput. Neurosci. 12, 9 (2018).
[11] S. Ostojic, Nat. Neurosci. 17, 594 (2014).

[12] C. van Vreeswijk and H. Sompolinsky, Science 274, 1724 (1996).

[13] M. Volgushev, I. Kudryashov, M. Chistiakova, M. Mukovski, J. Niesmann, and U. T. Eysel, J. Neurophysiol. 92, 212 (2004).

[14] A. Manwani and C. Koch, Neural Comput. 13, 1 (2001).

[15] L. A. Gatys, A. S. Ecker, T. Tchumatchenko, and M. Bethge, Phys. Rev. E 91, 062707 (2015).

[16] M. Mézard, G. Parisi, and M. Virasoro, Spin Glass Theory and Beyond: An Introduction to the Replica Method and its Applications (World Scientific Publishing Co. Inc., Singapore, 1987), Vol. 9.

[17] R. Engelken, F. Farkhooi, D. Hansel, C. van Vreeswijk, and F. Wolf, F1000Research 5, 2043 (2016).

[18] E. Ullner and A. Politi, Phys. Rev. X 6, 011015 (2016).

[19] A. Politi, A. Pikovsky, and E. Ullner, Eur. Phys. J. Spec. Top. 226, 1791 (2017). 
[20] W. Gerstner, W. M. Kistler, R. Naud, and L. Paninski, Neuronal Dynamics: From Single Neurons to Networks and Models of Cognition (Cambridge University Press, Cambridge, 2014).

[21] D. Golomb, Scholarpedia 2, 1347 (2007).

[22] M. Mattia and P. D. Giudice, Neural Comput. 12, 2305 (2000).

[23] R. Brette, M. Rudolph, T. Carnevale, M. Hines, D. Beeman, J. M. Bower, M. Diesmann, A. Morrison, P. H. Goodman, F. C. Harris, M. Zirpe, T. Natschläger, D. Pecevski, B. Ermentrout, M. Djurfeldt, A. Lansner, O. Rochel, T. Vieville, E. Muller, A. P. Davison, S. El Boustani, and A. Destexhe, J. Comput. Neurosci. 23, 349 (2007).

[24] One should also remember that because of the refractory period, $T>\tau_{R}$.

[25] S. Ostojic, J. Neurophysiol. 106, 361 (2011).

[26] Here and everywhere power spectra are represented, they are normalized in such a way that the total power is obtained by integrating over all positive frequencies: $0 \leqslant f<+\infty$.

[27] J. Friedrich and W. Kinzel, J. Comput. Neurosci. 27, 65 (2009).

[28] B. Lindner, Phys. Rev. E 73, 022901 (2006).

[29] In finite networks, sample-to-sample fluctuations are expected. Simulations of five different networks show that the standard deviation of $\langle v\rangle$ is $\sigma_{v} \approx 0.4$, while that of $C_{v}$ is $\sigma_{C}=0.01$.
Additionally, one expects the single steps of the recursive procedure are affected by statistical fluctuations: We have verified that the uncertainty of $\langle v\rangle$ is about 0.05 , while that of $C_{v}$ is approximately 0.005 .

[30] T. Schwalger and L. Schimansky-Geier, Phys. Rev. E 77, 031914 (2008).

[31] No appreciable differences can be noticed while referring to the following iterates.

[32] Leaving aside the zero-frequency channel which contributes to the average and is treated differently.

[33] The quantitative differences with the results for $P_{s}$ reported in Ref. [10] are quite likely to be attributed to the lack of accuracy in the integration scheme employed therein.

[34] R. Moreno-Bote and N. Parga, Phys. Rev. Lett. 92, 028102 (2004).

[35] N. Fourcaud-Trocmé, D. Hansel, C. Van Vreeswijk, and N. Brunel, J. Neurosci. 23, 11628 (2003).

[36] L. Badel, S. Lefort, R. Brette, C. C. Petersen, W. Gerstner, and M. J. Richardson, J. Neurophysiol. 99, 656 (2008).

[37] Y. Tsubo, Y. Isomura, and T. Fukai, PLoS Comput. Biol. 8, e1002461 (2012).

[38] S. Vellmer and B. Lindner, Phys. Rev. Res. 1, 023024 (2019). 